\title{
Research on Random Flow Simulation Model of Bridges
}

\author{
Li Cao and Limin Sun \\ Department of Bridge Engineering, Tongji University, Shanghai, China
}

\begin{abstract}
With the rapid development of automobile industry and economy, the randomness of traffic flow characteristics such as vehicle speed and vehicle weight acting on the bridge is more and more obvious. However, the previous studies are based on determination of traffic flow, so fatigue, deflection and other issues of bridges will be deviated from actual situations. Therefore, the model fitting the vehicle characteristics such as vehicle speed, vehicle weight, headway, model proportion and so on is found based on the statistical characteristics of the traffic flow on the actual bridge in this paper. The parameters of the model are statistically determined and the traffic flow program based on Monte-Carlo algorithm is established. Finally, the results show that the above theory can well simulate the actual traffic flow of the bridge through comparing the results of the program with the statistics of an actual bridge.
\end{abstract}

Keywords-traffic flow; MATLAB; time interval

\section{INTRODUCTION}

Because of the complexity of the traffic flow on the bridge, it is necessary for us to study the traffic flow characteristics of the bridge, to establish a scientific traffic flow model, to accurately describe and predict traffic flow on the bridge, and to provide science data support for subsequent bridge fatigue, deflection problems and so on. For the traffic flow model, there are mainly macroscopic continuous flow model [1], mesoscopic gas dynamics model and microscopic following model [2], and cellular automata model [3]. Some scholars have proposed that the distribution of expressway traffic capacity obeys lognormal distribution and Gamma distribution [4]. The literature [5] developed a set of traffic flow generating procedure based on Monte-Carlo algorithm, combined VIS-SIM simulation software, and realized real-time simulation of traffic flow in different states. In literature [6], the random traffic flow program of each lane through Monte-Carlo algorithm was established based on the traffic flow measured by toll and weighing system on the Nanjing Yangtze River Third Bridge. In literature [7], a traffic jam reconstruction model based on sequential Monte-Carlo method was proposed to evaluate current road conditions and reasonably estimate the congestion situations. The existing literature mainly studies traffic flow characteristics of the entire traffic line. In this paper, a traffic flow simulation model of the bridge is established according to the statistical characteristics of the traffic flow of the bridge.

\section{THEORY}

First of all, investigation and statistics on the traffic flow characteristics of specific bridges is conducted, which determines the distribution model that accurately reflects the characteristics of vehicle weight, lateral position, vehicle speed and headway in a random traffic flow, and determines the parameters of the corresponding model. A bridge random flow simulation model based on Monte-Carlo algorithm [8] through MATLAB is developed. The flow chart is shown in Figure 1 below.
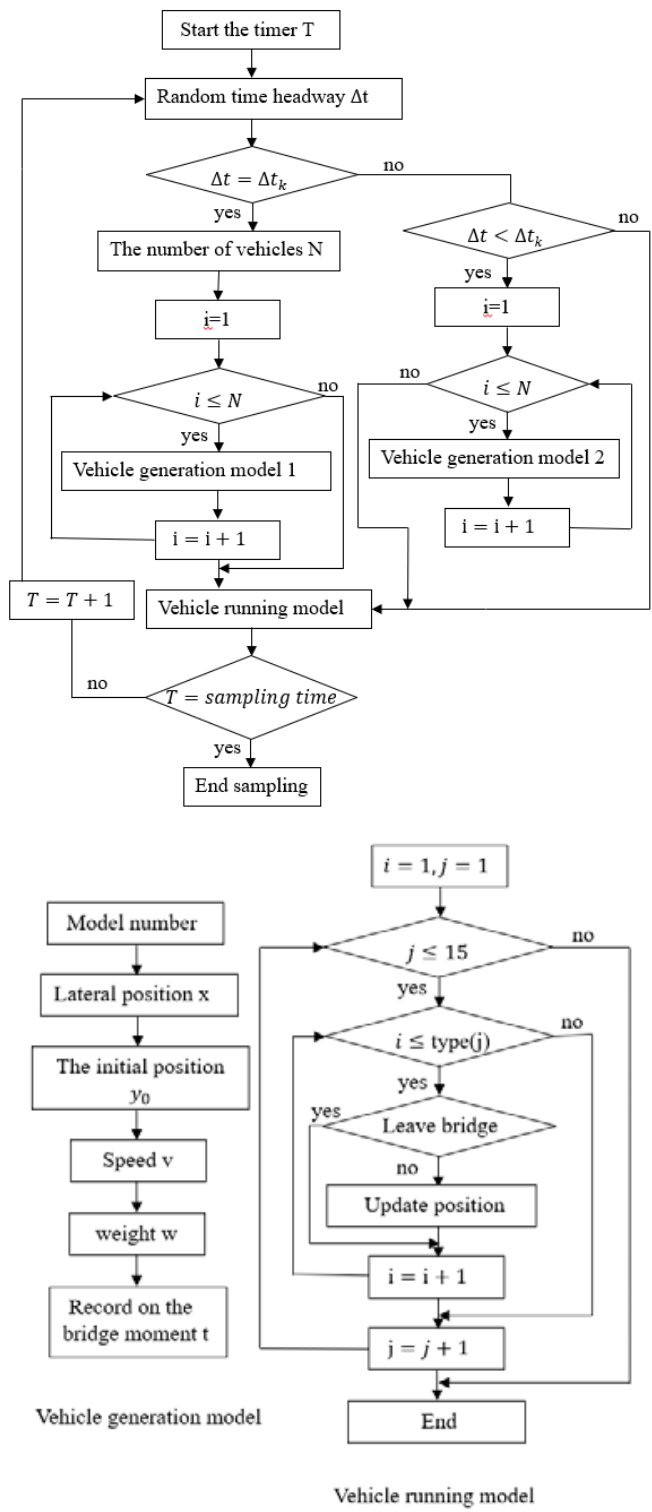

FIGURE I. FLOW CHART OF RANDOM FLOW SIMULATION MODEL 
Among them, the random time interval is generated based on the Monte-Carlo algorithm, that is to produce a uniform random vector between 0 and 1, which is represented as the probability of the time interval, and then the time interval $\Delta t$ is obtained through finding the inverse function of the probability distribution function. Suppose the time interval distribution function is:

$$
F(x=k)=\left\{\begin{array}{cl}
1-e^{-\left(\frac{x=k}{\eta}\right)^{m}} & , x \geq 0 \\
0 & , x<0
\end{array}\right.
$$

Where, $X$ represents the time interval of two adjacent cars, $F(x=k)$ represents the probability as the time interval $x$ of two adjacent cars is $k$.

$$
x=\eta \sqrt[m]{-\ln (1-F(x=k)}
$$

A random variable $x$ can be obtained by Eq (2) by assuming probability $F(x=k)$ as a uniform random vector within $(0,1)$.

\section{Finite ELEMENT Simulation}

The simulation of random vehicle is based on the parameters such as vehicle weight, lateral position, vehicle speed and time interval of vehicles in random traffic flow provided in reference [9], which reckons that the vehicle speed follows the normal distribution, the time intervals follows Wilbur distribution, vehicle weight obeys the logarithm normal distribution, the vehicle lateral position obeys the normal distribution. The vehicle weight, speed, lateral position and time interval and other data of the random traffic flow in one day are extracted through running random traffic simulation model, which is compared with the measured data.
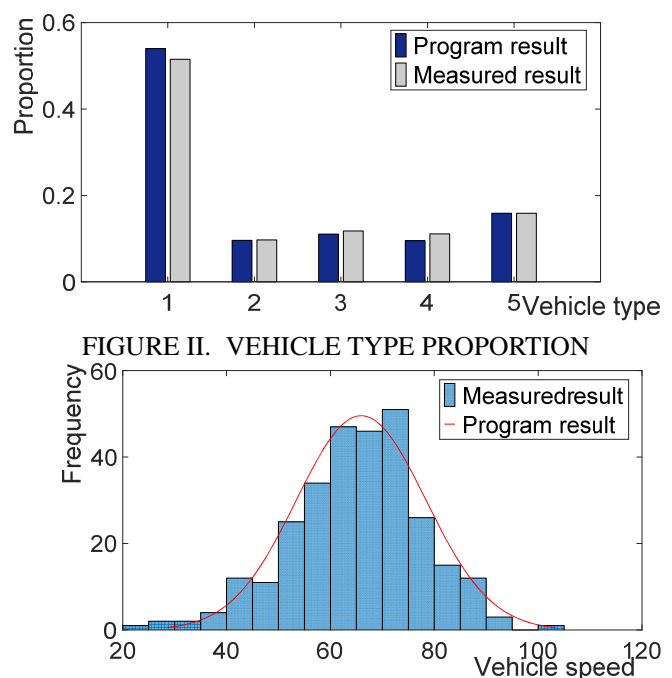

FIGURE III. FOURTH VEHICLE SPEED PROFILE

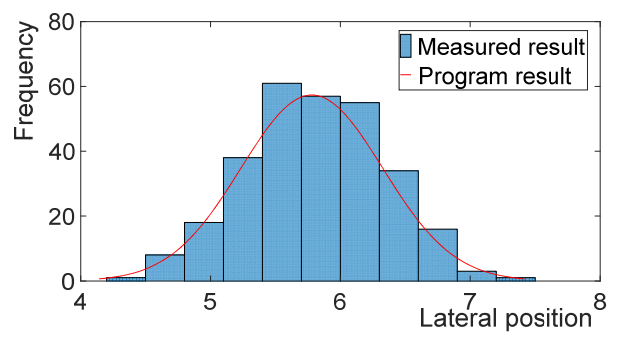

FIGURE IV. FOURTH VEHICLE LATERAL POSITION

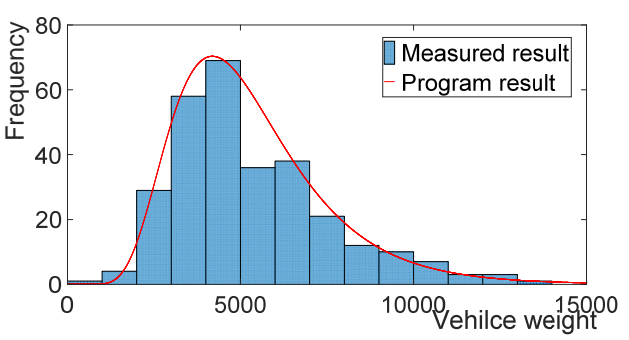

FIGURE V. FOURTH VEHICLE WEIGHT

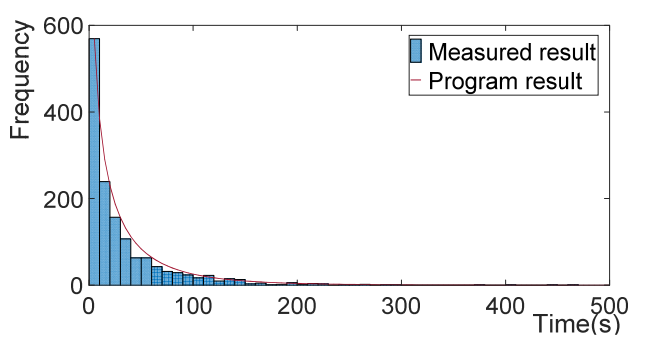

FIGURE VI. TIME INTERVAL DISTRIBUTION

\section{CONCLUSION}

The traffic flow on the bridge is a stochastic process. The traffic flow characteristics such as vehicle model proportion, vehicle speed, lateral position, vehicle weight and time interval follow a certain probability distribution. The traffic flow data of the actual bridge are used to determine the traffic flow distribution types and parameters. Then the Monte-Carlo method is used to generate a uniform random number and the inverse function of the distribution function is used to simulate the traffic flow characteristics. It is known that the speed follows the normal distribution, the time interval follows the Wilbur distribution, the vehicle weight follows the logarithm normal distribution, the vehicle lateral position follows the normal distribution according to the bridge traffic flow survey data. As shown in the above Figure II to Figure VI, the adopted random traffic flow model can well reflect the actual vehicle model characteristics such as proportion, speed, lateral position, vehicle weight and time interval.

\section{REFERENCES}

[1] Peng, G.H., et al., A new lattice model of traffic flow with the consideration of the traffic interruption probability. Physica A Statistical Mechanics \& Its Applications, 2012. 391(3): p. 656-663.

[2] Gazis, D.C., R. Herman, and R.W. Rothery, Nonlinear Follow-TheLeader Models of Traffic Flow. Operations Research, 1961. 9(4): p. 545567.

[3] Cremer, M. and J. Ludwig, A fast simulation model for traffic flow on the basis of Boolean operations. Mathematics and Computers in Simulation, 1986. 28(4): p. 297-303. 
[4] Xing, J., et al., Analysis of Bottleneck Capacity and Traffic Safety in Japanese Expressway Work Zones. in Transportation Research Board 89th Annual Meeting. 2010.

[5] ZHENG Xiao-yan, et al., Traffic flow simulation based on Monte-Carlo algorithm. Journal of Hefei University of Technology: Natural Science Edition, 2009. 32(9): p. 1374-1377.

[6] XU Han-jiang, et al., Vehicle Load Analysis of the Third Nanjing Yangtze River Bridge Based on Measured Traffic Flow. Journal of Disaster Prevention and Mitigation Engineering, 2011, 31(Supplement): p. 48-51.

[7] Huang Yu-da, WEI Xia, and WANG Yi-ran, A Sequential Monte Carlo Method-based Event Reconstruction Method for Traffic Event Simulation. Computing Technology and Automation, 2017(1): p. 150-154.

[8] HUANG Ling, The interval estimation of Multi-parameter distribution based on the Monte Carlo method. 2016, Central China Normal University.

[9] GAO Wen-bo, Theory and Program Development of Highway Traffic Flow Simulation Based on Measured Data. 2010, Chang'an University. 